\title{
LOS IMPERFECTOS DE SUBJUNTIVO Y LA REESTRUCTURACIÓN DEL SISTEMA VERBAL ESPAÑOL A FINALES DEL SIGLO DE ORO
}

\begin{abstract}
Luquet Gilles, Los imperfectos de subjuntivo y la reestructuración del sistema verbal español a finales del siglo de oro [The subjunctive imperfect tense and the restructuring of the Spanish verb system towards the end of the Spanish Golden Age]. Studia Romanica Posnaniensia, Adam Mickiewicz. University Press, Poznań, vol. XXXI: 2004, pp. 361-368. ISBN 83-232-1353-4, ISSN 0137-2475.
\end{abstract}

\begin{abstract}
A historical analysis of the Spanish verb shows that the transition between the classical system and the modern system is characterized by two disappearances: the future subjunctive and the contrast between cantara and cantose. The present paper aims to show that: 1) abandoning the use of the future subjunctive has led ditectly to abandoning the contrast between cantara and cantase; 2) by the end of the classical period cantara was a form which was "programmed" to replace cantase; 3) in the modern and contemporary verbal system, cantara remains the same as in the classical system. Even if its referential capacitics have somewhat altered, it remains the same single linguistic sign. This paper is based on a modal theory which differs the traditional contrast between indicative an subjunctive.
\end{abstract}

Para un especialista en literatura española, los siglos XVI y XVII representan el período en que se observa el paso -por decirlo en términos reductores- de la naturalidad clásica a la artificiosidad barroca, de la ponderación a la exageración, de la simplicidad a la complicación. Para un lingüista, al contrario, el mismo período se caracteriza por una singular simplificación del idioma. Uno de los ejemplos más evidentes de este fenómeno es el que se observa en el ámbito fonológico -va disminuyendo el número de fonemas con el que el castellano construye sus significantes- pero se simplifica también la morfología del idioma en la medida en que algunos de sus morfemas gramaticales desaparecen o dejan de oponerse entre sí. Las consideraciones que siguen serán relativas a una de las manifestaciones de este fenómeno en la organización del sistema verbal.

Hablar del verbo español es hablar de formas lingüísticas cuyo contenido se define en el marco de varios sistemas de representaciones. En el verbo, concretamente, existen varias representaciones posibles del tiempo implicado por una operación y varias representaciones posibles del ser con el que se relaciona esta 
operación. Existe, en otros términos, todo lo que permite describir este conjunto de signos como un sistema de modos, de "tiempos" -en el sentido gramatical de la palabra- $y$ de personas.

En el verbo del español del siglo XVI se oponían dos modos personales:

- un modo actualizador, que constaba de un presente, un pretérito y un futuro (canto, canté, cantaré);

- un modo inactualizador, que constaba de unidades de representación mucho más numerosas (cantaba, cantaría, cante, cantare, cantara y cantase).

Huelga decir que esta descripción no es la de la tradición gramatical. Para más informaciones sobre sus fundamentos teóricos pueden verse los últimos capítulos de G. Luquet, Regards sur le signifiant. Etudes de morphosyntaxe espagnole (Paris, Presses de la Sorbonne Nouvelle, 2000) y sobre todo, del mismo autor, una monografía de próxima aparición: La teoría de los modos en la descripción del verbo español. Un nuevo planteamiento.

Lo que importa destacar aquí es que, en el marco teórico en que se sitúan los presentes análisis, las llamadas formas «subjuntivas» del verbo pertenecen al mismo modo que las del tipo cantaba y cantaría y se caracterizan por una temporalidad muy distinta de la que corresponde a las formas actualizadoras: la representación del tiempo que conllevan las formas cante y cantare es un presentefuturo indiferenciado (es decir, un espacio simplemente no pretérito); la representación del tiempo que conllevan cantara y cantase es de tipo más indeterminado todavía. Los acontecimientos expresados por los imperfectos de subjuntivo de la lengua clásica podían ir asociados -como hoy en día- al pasado, al presente o al futuro del enunciador.

Estos dos imperfectos, desde luego, no eran equivalentes en el sistema, y tampoco había equivalencia entre presente y futuro de subjuntivo. La oposición entre cante y cantare, por una parte, y la oposición entre cantara y cantase, por otra parte, eran dos características esenciales del sistema verbal clásico y basta con abrir al azar una obra literaria del siglo XVI o de principios del XVII para comprobar que los autores de ese período las explotaban frecuentemente y conseguían con ellas los efectos discursivos más sutiles. Si se toma en cuenta lo que era el rendimiento funcional de estas oposiciones, incluso puede parecer sorprendente que estuvieran condenadas a desaparecer antes de que terminara la Edad de Oro de la literatura española, pero los textos mismos no dejan lugar a duda: hacia finales del primer tercio del siglo XVII, el futuro de subjuntivo se estaba convirtiendo en una forma lingüística moribunda $y$, por otra parte, se estaba generalizando la tendencia a emplear cantara en vez de cantase, o sea, la tendencia a renunciar a la oposición de estas dos formas. A finales del siglo XVII, las formas personales del verbo español seguían repartiéndose entre dos modos, pero si uno de ellos -el modo actualizadorseguía idéntico a lo que era dos siglos antes, el otro -el modo inactualizador- había perdido ya para muchos hablantes dos de sus seis unidades de representación.

Este fenómeno es el que caracteriza la evolución del verbo español durante el período clásico de su historia y es su carácter sistemático lo que se pretende sub- 
rayar aquí. Se tratará de mostrar concretamente que la desaparición de la oposición cantara / cantase no es más que una consecuencia de la desaparición del futuro de subjuntivo.

Antes, sin embargo, conviene dar algunas precisiones sobre la fecha de estas desapariciones, ya que la mayoría de los escritores del período clásico explotan en sus obras un modo inactualizador que comprende todavía cuatro formas de tipo subjuntivo.

Por lo que se refiere al futuro de subjuntivo, por ejemplo, hay que esperar la década 1630-1640 para observar que su frecuencia de empleo, en las obras literarias, disminuye de manera considerable e incluso espectacular, una manera que anuncia, a más o menos largo plazo, el abandono definitivo de esta forma verbal. Las cifras que contienen los trabajos de L.O. Wright, por ejemplo, lo muestran sin ambiguiedad $^{1}$. Pero esta visión del fenómeno, basada en la observación del habla escrita y literaria, no proporciona ninguna información sobre el lugar que ocupaba el futuro de subjuntivo en el español que se hablaba en la Península a principios del siglo XVII. Y según lo muestran todos los trabajos realizados sobre el tema ${ }^{2}$ hay motivos para pensar que en la lengua oral -por lo menos en determinados niveles sociolinguísticos- cantare es una forma que estaba ya en vías de desaparición a principios del siglo XVI e incluso a finales del siglo XV. Lo que se observa en la lengua escrita del siglo XVII no es pues un fenómeno nuevo, sino la manifestación objetiva -y tardía- de una tendencia cuyos orígenes son probablemente mucho más antiguos: la tendencia a eliminar de las representaciones del verbo la que corresponde al futuro de subjuntivo ${ }^{3}$. Lo que sí se comprueba sin dificultad es que el futuro de subjuntivo castellano se convierte en un arcaísmo lingiístico en los siglos XVIII y XIX y que, en el siglo XX, no existe más que como vestigio de un estado de lengua anterior ${ }^{4}$.

${ }^{1}$ L. O. Wright (1932), The -Ra Verb Form in Spain. Véase sobre todo el Apéndice B.

${ }^{2}$ Véanse, entre otros, G. Luquet (1988a), Sobre la desaparición del futuro de subjuntivo en la lengua hablada de principios del siglo XVI; R. Eberenz (1990), "Sea como fuere». En torno a la historia del futuro de subjuntivo español; G. de Granda Gutiérrez (1968), Formas en «-re» en el español atlántico y problemas conexos.

${ }^{3}$ Las primeras manifestaciones de esta tendencia se remontan aparentemente a los últimos años del siglo XIII -cuando el presente de subjuntivo empezó a sustituir a cantare en las oraciones temporales introducidas por después que- y se multiplicaron luego de manera continua. Para algunas indicaciones al repecto, véase G. Luquet (1988b), Systématique historique du mode subjonctif espagnol, p. $165-169$ y $243-245$.

${ }^{4}$ Entre las causas de la desaparición de cantare figuran probablemente las propiedades que hacían de él una excepción en su propio sistema:

- De todas las formas predicatjvas del verbo era la única que no podía constituir el núcleo de una oración independiente o principal (no se prestaba más que a la expresión de determinados tipos de subordinación).

- De todas las formas simples del verbo era la única que asociaba la representación de un acontecimiento a la anterioridad obligada de otro. 
El abandono de la oposición entre cantara y cantase -más tardío que el abandono de cantare - es algo que sólo empieza a manifestarse a finales del siglo XVI y a principios del XVI. Recordaré aquí que desde finales del siglo XV, cantara y cantase eran unidades de representación que no se oponían por su temporalidad sino por su manera de inactualizar la representación de un acontecimiento. Cantara, concretamente, era una forma inactualizadora marcada una forma que, en la práctica discursiva, tenía la particularidad de asociar la representación de un acontecimiento al ámbito nocional de lo «irreal», es decir, el ámbito de lo irrealizado o de lo irrealizable-, mientras que cantase, forma no marcada, era la que se usaba para la expresión de lo posible, de lo que era objeto de deseo, de duda, de apreciación subjetiva, etc. Es lo que aparece, por ejemplo, en este pasaje de la Diana de Montemayor, en el que se oponen -pese a las apariencias- dos maneras de concebir una hipótesis:

- Dime, Sireno, si acaso viesses la hermosa Diana con su esposo y estar los dos con todo el contentamiento del mundo, riéndose de los amores que tú con ella avías tenido, ¿qué harias? Sireno respondi6:

- Por cierto, señora, ninguna pena me darían, antes les ayudaría a reyr de mis locuras passadas.

- Felicia replicó:

- Y si acaso ella fuera agora soltera y se quisiera casar con Sylvano y no contigo, ¿qué hizieras?

- Sireno respondio:

- Yo mismo fuera el que tratara de concertallo.

Lo que se expresa en el primer esquema condicional (si vieses la hermosa Diana ... ¿qué harías?) es una hipótesis irreal presente. Sireno no está presenciando la escena descrita por Felicia, pero suponer lo contrario no es concebir una hipótesis descabellada. Lo supuesto es algo imaginable y eso es lo que expresa el uso del subjuntivo en -se en la prótasis del enunciado. Lo que se expresa en la segunda estructura condicional (si ella fuera soltera ... ¿qué hizieras?) es otra hipótesis irreal y presente, pero lo que se supone entonces se opone no sólo a la realidad contextual -Diana no es soltera- sino también a lo que se ha dicho en la primera estructura condicional, ya que entonces se aludía no sólo a Diana sino también a su esposo. La hipótesis se concibe en el campo de lo irrealizable y eso es lo que expresa el uso del subjuntivo en -ra en todo el enunciado.

El valor de cantara, en el sistema verbal clásico, empieza a cambiar cuando aparece en enunciados del tipo siguiente:

Diole [a mi amo] tanta gana de reír en verme de aquella manera que llamó a los que con él jugaban para que me vieran. Riéronse todos y rogaron que [...] se me perdonase.

${ }^{5}$ J. de Montemayor, Los siete Libros de la Diana, Madrid, Espasa-Calpe, 1970, p. 225 (ejemplo citado -con otra interpretación- en I. Andrés Suárez, El verbo español. Sistemas medievales y sistema clásico, Madrid, Gredos, 1994, p. 158). 
Vieran, en semejante contexto, expresa un acontecimiento virtual que no se concibe en el campo nocional de lo irrealizable. Esta forma verbal no es sino un mero sustituto de viese, es decir el sustituto de un imperfecto de subjuntivo de tipo no marcado. Estamos en 1499: el personaje que se expresa así se llama Guzmán de Alfarache y con él empieza el largo proceso de equiparación de cantara a cantase.

¿Por qué empezaron a equipararse estas dos formas verbales a finales del siglo XVI? Cabe pensar que fue probablemente por varios motivos y que uno de ellos, por lo menos, fue de tipo sistemático. Uno de ellos tendría que ver con la tendencia -afirmada ya desde el principio del período renacentista- a eliminar del sistema el futuro de subjuntivo.

Lo que importa observar, efectivamente, es que durante todo el siglo XVI, la incipiente eliminación de cantare afectó directamente al equilibio interno del modo inactualizador y, más concretamente, al equilibrio interno del subsistema subjuntivo. En la medida en que ese subsistema constaba de dos formas asociadas a un tiempo de tipo 'presente-futuro' (cante, cantare) y otras dos asociadas a un tiempo de tipo indiviso (cantara, cantase) no cuesta mucho admitir que la desaparición de uno de los miembros del primer par iba a acarrear la desaparición de uno de los miembros del segundo. Se trataba sencillamente de una exigencia de pura coherencia interna, una exigencia sistemática.

Esta exigencia tiene probablemente algo que ver con el hecho de que Mateo Alemán y algunos de sus contemporáneos empezaran a ver en cantara una forma capaz de hacer las veces de cantase. Tiene probablemente algo que ver con el hecho de que el modo inactualizador que intentó imponerse en la lengua a partir del siglo XVII no conservara más que cuatro de las seis unidades de representación que tenía anteriormente:

modo inactualizador

medieval y clásico

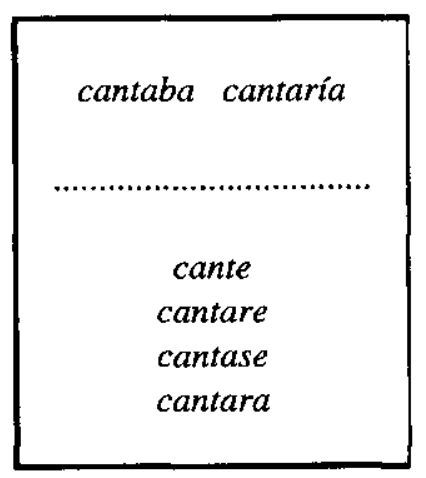

modo inactualizador en construcción en la lengua postclásica

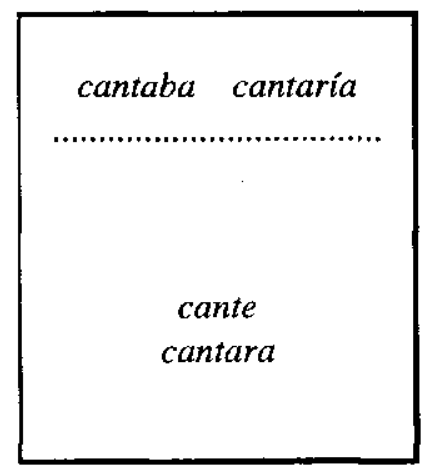


Lo único que podía dificultar la generalización del nuevo sistema -lo único que de hecho la dificultó y la sigue dificultando hoy en día- era el problema que planteaba su organización semiológica, o sea, el que representaba la eliminación de uno de los dos antiguos imperfectos de subjuntivo.

Esta eliminación, efectivamente, no podía ser tan «fácil» como la del futuro de subjuntivo por dos motivos:

- En el sistema que intentaba imponerse en la lengua a finales del siglo XVII y más concretamente en el modo inactualizador de dicho sistema- ya no existía la representación a la que remitía, en el sistema medieval y clásico, el futuro de subjuntivo. Ya no existía la representación preconstruida de un acontecimiento situado, por una parte, en la anterioridad de otro y, por otra parte, en un 'presentefuturo' inactualizador. Cantare, por lo tanto, estaba condenado a desaparecer y sólo el carácter conservador de la lengua escrita y literaria podía frenar -como hizo efectivamente durante dos siglos- esta desaparición programada.

- En el mismo sistema, en cambio, seguía existiendo la representación a la que remitía, en la lengua medieval y clásica, un imperfecto de subjuntivo. Seguía existiendo la representación de un acontecimiento concebido fuera de la actualidad y asociado a la imagen de un tiempo indiviso.

Seguía existiendo pues una forma cuyo significado era más abstracto que el de cualquier otra forma inactualizadora del sistema:

- más abstracto que el de cantaba o de cantaría, ya que una forma que conlleva la representación de un tiempo indiviso es más abstracta que la que conlleva la representación de un presente inactualizado (cantaba) o la que conlleva la representación de un futuro inactualizado (cantaría) ${ }^{6}$;

- más abstracto que el de cante, ya que una forma que conlleva la representación de un tiempo indiviso es más abstracta que la que conlleva la representación de un tiempo de tipo 'presente-futuro'.

En la nueva estructura, por decirlo con otras palabras, seguía existiendo una forma dotada de una capacidad de inactualización sin equivalente en el sistema, una forma inactualizadora claramente marcada.

Ahora bien, hablar de una forma inactualizadora marcada asociada a la representación de un tiempo indiviso es hablar de una forma cuyo significado es exactamente el de la forma en -ra del castellano clásico. Es hablar de una forma que tiene las mismas propiedades intrínsecas y la misma propiedad distintiva, es decir, el mismo valor. La única diferencia es que este valor, en el nuevo sistema, había de determinarse por oposición a tres términos en vez de cinco.

El imperfecto de subjuntivo con el que tenía que construirse el modo inactualizador, en la lengua postclásica, era pues el imperfecto en -ra y, de hecho, fue lo

\footnotetext{
${ }^{6}$ Para la defjnición de cantaba y cantaría en la teoría del verbo utilizada aquí, véase G. Luquet
} $(2000)$, p. 91-103. 
que intuyeron quienes empezaron, como Mateo Alemán, a renunciar a la oposición entre cantara y cantase. Lo hicieron usando cantara en vez cantase y no lo contrario?.

En la práctica, sin embargo, era difícil que desapareciera rápidamente una oposición de tan alto rendimiento funcional como la de los dos imperfectos de subjuntivo de la lengua clásica. De hecho, cuatro siglos más tarde, no ha desaparecido todavía:

- es cierto que cantara y cantase, en la lengua de hoy, son «intercambiables» en una inmensa mayoría de casos;

- es cierto que cantase tiene una frecuencia de empleo globalmente muy inferior a la de cantara ${ }^{8}$;

- pero no es menos cierto que cantara sigue conservando aptitudes referenciales que no comparte con cantase («quisiera pedirte un favor», y no "*quisiese pedirte un favor», «pudieras [o debieras] tener más cuidado», y no «*pudieses [0 *debieses] tener más cuidado»).

Conviene admitir, por lo tanto:

- que la reestructuración del sistema verbal -iniciada en el Siglo de Oro- no ha terminado todavía;

- que siguen existiendo, en el modo inactualizador de dicho sistema, dos imperfectos de subjuntivo';

- que uno de ellos, cantara, tiene una capacidad de inactualización superior a la del otro, como en el Siglo de Oro:

${ }^{7}$ Véase G. Luquet (1988b), p. 263-269.

${ }^{8}$ En 1971, Vidal Lamíquiz consideraba ya que en la lengua oral y en la lengua periodistica, cantara se usaba dos veces más que cantase (Cantara y cantase, p. 3-4).

${ }^{9}$ Dos imperfectos de subjuntivo a cuyo significado de lengua, desde luego, no tienen acceso los hablantes. En un artículo en el que intenta describir las propiedades respectivas de las dos formas (Subjunctive -RA and -SE: Free Variation?), Dwight Bolinger, en 1956, resume el sentimiento lingüístico de su informante castellano diciendo que la forma en -se, para él, implica distanciación, indiferencia, hipótesis, imprecisión, mayor inverosimilitud («remoteness, detachment, hypothesis, lack of interest, vagueness, greater unlikelihood»), en oposición a la forma en - ra que implica a menudo una imagen «relativamente» más neta del acontecimiento expresado ( $«$-ra brings everything into relatively sharper focus»). En 1969, en cambio, Vidal Lamiquiz, en «El sistema verbal del español actual: intento de estructuración", considera que la oposición entre las dos formas se argumenta de la manera siguiente: «inclinación subjetiva hacia la imposibilidad» $(-r a)$ / «inclinación subjetiva hacia la posibilidad» (-se) y lo ilustra considerando que en un enunciado como le ordenó que viniese inmediatamente la acción verbal, "dentro de la no realización que supone el subjuntivo, se ve con grandes posibilidades de cumplirse", mientras que en le ordenó que estudiara "captamos poco convencimiento de que [la] orden se cumpla». La contradicción no puede ser más evidente: la mayor distanciación con relación a la actualidad, o sea, la mayor capacidad de inactualización, es una propiedad de la forma en -se para el informante de Dwight Bolinger y de la forma en -ra para Vidal Lamíquiz. 


\section{estructura del modo inactualizador \\ en la lengua de hoy}

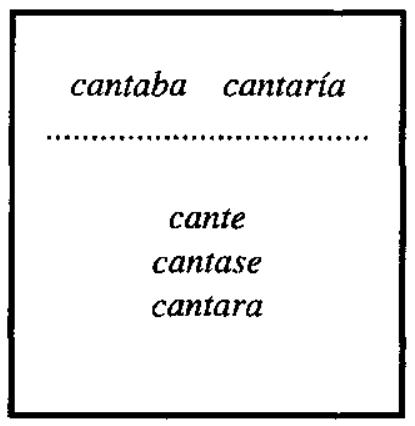

Si se añade a esto que el cantara de hoy conlleva la misma representación del tiempo lingüístico que el cantara clásico -la de tipo indiviso- y que el cantara clásico conllevaba ya la misma representación del tiempo lingüístico que el cantara medieval ${ }^{10}$, no hay motivo para considerarlos como signos distintos. Hablar de una forma que ha pertenecido siempre al mismo modo, que ha sido siempre la forma marcada de dicho modo y que ha estado asociada siempre a la misma representación del tiempo lingüístico, es hablar de un mismo signo. Es hablar de un mismo signo y de sus distintas capacidades referenciales. De una misma herramienta linguística usada de distintas maneras en distintas épocas.

\section{REFERENCLAS BIBLIOGRÁFICAS}

Andrés Suárez, I. (1994), El verbo español. Sistemas medievales y clásico. Madrid: Gredos.

Bolinger, D. L. (1956), Subjunctive -RA and -SE: Free Variation?, Hispania, XXXIX, 372-373.

Eberenz, R. (1990), «Sea como fuere». En tomo a la historia del futuro de subjuntivo español, en

I. Bosque (ed.), Indicativo y subjuntivo. Madrid: Taurus, 383-409.

Granda Gutiérrez, G. de (1968), Formas en «-re» en el español atlóntico y problemas conexos,

Thesaurus, Boletín del Instituto Caro y Cuervo, XXIII (enero-abril) 1, 1-22.

Lamíquiz, V. (1969), El sistema verbal del español: intento de estructuración, Revista de la

Universidad de Madrid, XVII, 241-265.

- (1971), Cantara y cantase, Revista Española de Lingüística, 1, 1, 89-96.

Luquet, G. (1988a), Sobre la desaparición del futuro de subjuntivo en la lengua hablada de prin.

cipios del siglo XVI, en M. Ariza et alii (eds.), Actas del I Congreso Internacional de Historia de

la Lengua Española (Cáceres, 30 de marzo - 4 de abril de 1987). Madrid: Arco/Libros, 509-514.

- (1988b), Systématique historique du mode subjonctif espagnol. Paris: Klincksieck.

- (2000), Regards sur le signifiant. Etudes de morphosyntaxe espagnole. Paris: Presses de la Sorbonne

Nouvelle.

Wright, L. O. (1932), The «-ra» Verb Form in Spain. Berkeley: University of California Press.

${ }^{10}$ Véase G. Luquet (2000), p. 105-118. 